\title{
A Model for Nucleation and Growth Processes of Tin Whisker
}

\author{
Kiyomichi Nakai $^{1, a}$, Tatsuaki Sakamoto ${ }^{1, b}$, Sengo Kobayashi ${ }^{1, \mathrm{c}}$, \\ Kazuto Arakawa ${ }^{2, \mathrm{~d}}$, Hirotaro Mori ${ }^{2, \mathrm{e}}$, Masao Takamizawa ${ }^{3, \mathrm{f}}$, \\ Koji Murakami ${ }^{4, g}$ and Makoto Hino ${ }^{4, h}$ \\ ${ }^{1}$ Course of Materials Science and Engineering, Department of Materials Science and Biotechnology, \\ Graduate School of Science and Engineering, Ehime University, 3 Bunkyo-cho, Matsuyama \\ 790-8577, Japan \\ ${ }^{2}$ Research Center for Ultra-High Voltage Electron Microscopy, Osaka University, Mihogaoka, Ibaraki \\ 567-0047, Japan \\ ${ }^{3}$ OM Sangyo Co. Ltd., 3-18-48 Noda, Okayama 700-0971, Japan \\ ${ }^{4}$ Industrial Technology Center of Okayama Prefecture, 5301 Haga, Okayama 701-1296, Japan \\ ak-nakai@eng.ehime-u.ac.jp, 's sakamoto@eng.ehime-u.ac.jp, 'sengo@eng.ehime-u.ac.jp, \\ darakawak@uhvem.osaka-u.ac.jp, ${ }^{\mathrm{e}}$ mori@uhvem.osaka-u.ac.jp \\ takamiza@oms.co.jp, ${ }^{9}$ kouji murakami@pref.okayama.Ig.jp, ${ }^{\text {hmakoto hino@pref.okayama.jp }}$
}

Keywords: Bulk Sn, Edge and screw dislocations, Small-angle dislocation boundary, Pipe-diffusion, Atomic steps on surface, Amorphous oxide film,

\begin{abstract}
A model for the nucleation and growth processes of Sn whisker is offered. High density of localized screw dislocations by deformation form the dense spiral steps of atomic scale on Sn surface. The spiral steps would induce the nucleation of Sn whisker. Edge dislocations localized at the same region where the dense screw dislocations exist supply $\mathrm{Sn}$ atoms to $\mathrm{Sn}$ whisker through pipe diffusion. Both screw and edge dislocations would bend along almost one direction, namely, to relax the external shear stress. The image force also helps to bend the dislocations perpendicular to the whisker side-surface. The bending of dislocations at root of whisker leads the bend of whisker. The pipe diffusion of $\mathrm{Sn}$ atoms through edge dislocations from bulk Sn toward whisker is suppressed at the bent part of edge dislocation, resulting in release of Sn atoms inside whisker and leading to the growth of whisker near its root.
\end{abstract}

\section{Introduction}

Lead $(\mathrm{Pb})$ free electroplating has been attracted considerably because $\mathrm{Pb}$ tends to be eliminated from electronic devices according to the RoHS in EU in recent years. However, whisker forms remarkably under deformation in $\mathrm{Pb}$-free $\mathrm{Sn}$ electroplated layer, lowering reliability of electronic devices. The mechanism for the whisker formation has been investigated [1 - 10], but is still under discussions.

In the present investigation, the nucleation and growth mechanism of Sn whisker has been proposed based on the results obtained using bulk Sn.

\section{Experimental Procedures}

Two specimens of pure bulk Sn were cut into the parallelepiped shape of $15 \mathrm{~mm} \times 50 \mathrm{~mm} \times 3 \mathrm{~mm}$, and they were kept with and without tensile stress of around $15 \mathrm{MPa}$ for 20 days, respectively, using the tensile test equipment of Shimadzu Autograph AG-10kNIS. The microstructures of specimens 
were observed by an optical and a scanning electron microscopes. The ultra-high voltage transmission electron microscope (UHVEM, Hitachi H-3000) is used under an accelerating voltage of $2000 \mathrm{kV}$ for observing microstructures inside whisker in diameter around $3 \mu \mathrm{m}$ without thinning the whisker. The microstructures were recorded with video system.

\section{Results and Discussion}

Figure 1 shows nodular whiskers (encircled areas). The diameter of whisker is much smaller than grain size (average $\mathrm{Sn}$ grain size is $35 \mu \mathrm{m}$ ). It is characteristic that whiskers form mainly around grain boundaries. Figure 2 is the enlarged photos of Fig. 1, showing nodular whiskers. In the case of the Sn without the tensile stress, the number density of whiskers is greatly lower than that of the Sn with the tensile stress. It is obvious that the tensile stress enhances the formation of whiskers.

According to the above results, nucleation of whiskers would be affected by the inhomogeneity of the deformation, namely, heavy deformation occurs only inside each slip band (see Fig. 3, showing slip bands in strain-annealed Sn crystal, whose average grain size is 3 $\mathrm{mm})$. The deformation induces multiplication of dislocations and their conservative motions, resulting in their local accumulation around grain boundary. There exist remarkable slip bands on bulk Sn surface, and it could be suggested that high density of dislocations localized around the ends of slip bands at grain boundary consist of both screw and edge dislocations, because various directions of slip bands due to the operations of various slip systems accumulate around grain boundary. The motion of screw dislocations form

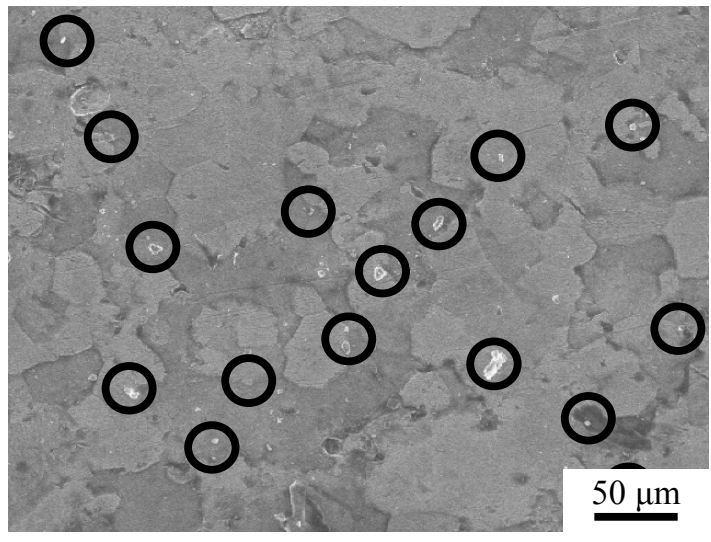

Fig. 1 Optical micrograph taken from the bulk Sn surface kept under tensile stress for 20 days at ambient temperature. Whiskers are encircled. steps on Sn surface in atomic scale (Fig. 4). The steps on Sn surface tend to trap $\mathrm{Sn}$ atoms and/or their aggregates migrating on Sn surface, because the step has combination sites with Sn atoms. Such process would form spiral steps around the end of screw dislocations at $\mathrm{Sn}$ surface. The height of a step is one atomic distance, and the spiral step continues to increase with increasing trapped $\mathrm{Sn}$ atoms [11,

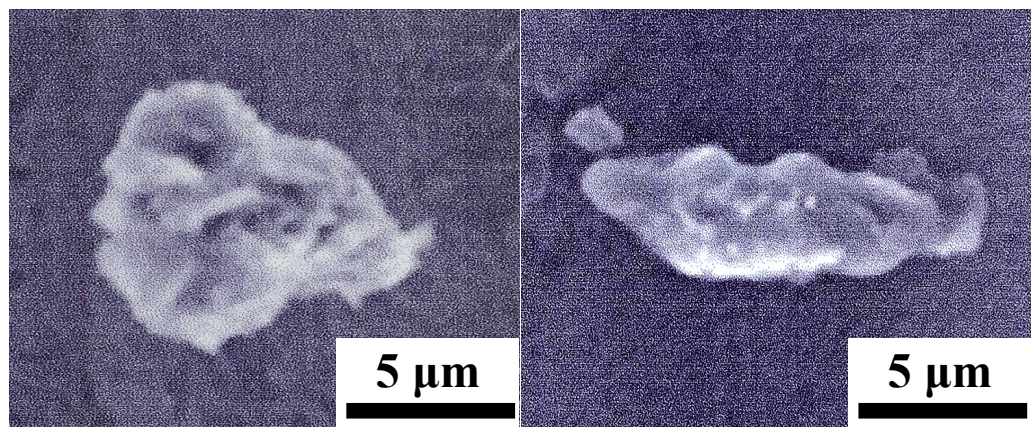

Fig. 2 Enlarged scanning electron micrographs taken from the nodular whiskers in Fig. 1.

12]. However, the spiral steps combine with each other when screw dislocations are densely localized by deformation $[11,12]$. When Sn atoms migrate into the valley between spiral steps (designated as "V" in Fig. 4), Sn atoms are easily combined with spiral steps, resulting in reduction of surface energy of spiral steps. The regions where screw dislocations are densely localized act as nucleation sites for whiskers because diffusing $\mathrm{Sn}$ atoms on crystal surface are preferably captured in the region. The shape of whiskers would depend on arrangements of high

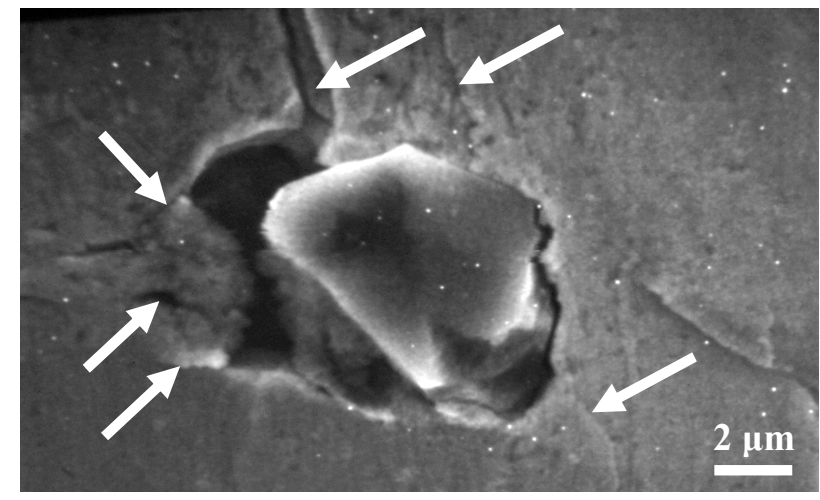

Fig. 3 Scanning electron micrograph of a whisker formed at the intersection of slip bands indicated by arrows. 
density of dislocations accumulated around grain boundaries. Some examples could be shown as follows. In the case of screw dislocations being densely localized and normal to the crystal surface [13], the height of step on surface due to the screw dislocations is the maximum (one atomic distance per a screw dislocation). Sn atoms migrating on the surface are easily trapped with high density of spiral steps, and

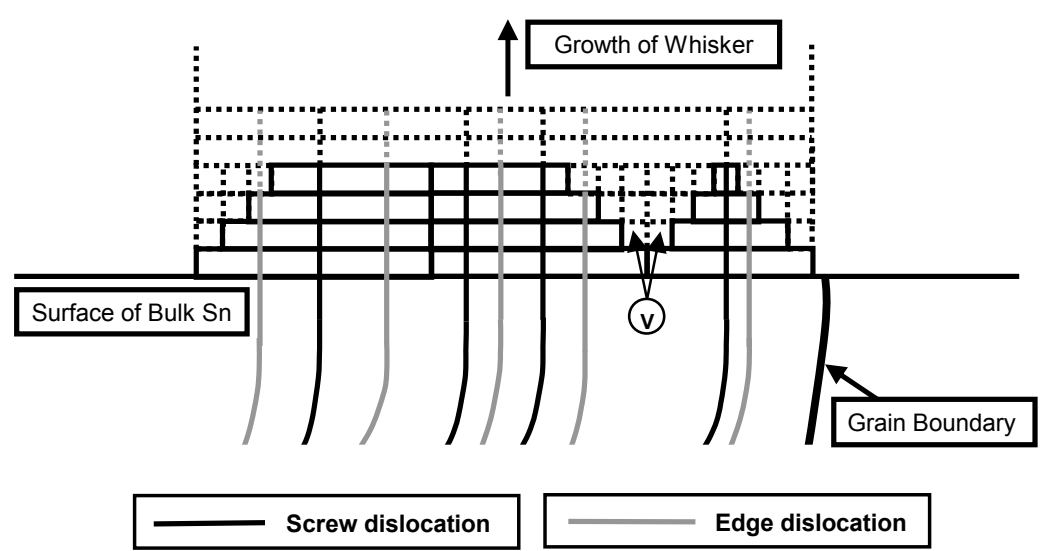

Fig. 4 Growth of whisker in association with screw and edge dislocations. would make whisker grow straight. On the other hand, in the case of a grain boundary inclining relatively to crystal surface, dislocations incline relatively to crystal surface under the applied tensile stress. In this case, the height of a step due to a screw dislocation is given by $|\boldsymbol{b}| \sin \theta$, where $|\boldsymbol{b}|$ is the size of Burgers vector and $\theta$ the angle between the Burgers vector and the bulk Sn surface. The height of step formed on the Sn surface is lower compared with the case that the screw dislocation is normal to the $\mathrm{Sn}$ surface. This would lead that $\mathrm{Sn}$ atom is difficult to be trapped with the step on crystal surface effectively. Then, the $\mathrm{Sn}$ atoms migrating on the surface tend to be captured in the wider region compared with that where screw dislocations are piled up normal to the surface by the external shear stress, resulting in the formation of nodular whisker [13]. It could be summarized that when the height of step on the surface due to screw component of dislocation is larger, the nucleation of straight whisker would be easily occur.

For the growth of whisker, it is indispensable that Sn atoms are supplied from the bulk Sn into whisker. The dense localization of edge dislocations at the same place where screw dislocations exist is also indispensable, because pipe diffusion of $\mathrm{Sn}$ atoms should occur along edge dislocations. The pipe diffusion of $\mathrm{Sn}$ atoms along edge dislocations facilitates the supply of $\mathrm{Sn}$ atoms from bulk $\mathrm{Sn}$ into whisker. In the case of low density of edge dislocations, growth rate of whisker would be low because it is difficult to supply Sn atoms. The whisker formation is then promoted in the case of both screw and edge dislocations being densely localized and normal to the surface. As shown in Fig. 3, whisker is nucleating at the location where many slip bands collide with each other and high density of both screw and edge dislocations would be piled up. In the case of Sn whose crystal structure is tetragonal, $(c / a)=0.55$, the edge dislocation is analyzed to have the Burgers vector of $\boldsymbol{b}=\langle 10 \overline{1}>$, which has been clarified to be easily multiplied on $\{101)$ planes [13]. The magnification of the $\boldsymbol{b}=$ $<10 \overline{1}>$ is relatively larger than others in $\mathrm{Sn}$, that is, the space neighbor to the extra half-plane of the edge dislocation is larger and the pipe diffusion of $\mathrm{Sn}$ atoms along the edge dislocation would be easily enhanced.

The growth process of whisker has been discussed. The straight whisker would bend during its growth. Whisker often bends at its root with or without the change in crystallographic orientation (C.O.). Just after the start of growth (nucleation) of whisker, both dense screw and edge dislocations piled-up in bulk Sn would be inherited into whisker, and high density of them are almost normal to the top surface of whisker. However, all the dislocations would move almost along the same direction as those in the bulk Sn, because the external shear stress on dislocations in the bulk Sn acts on those inside whisker. Furthermore, it could be suggested that stress would be free on the whisker surface. It is reasonable that dislocations with opposite sign of Burgers vectors (hereafter, referred to as image dislocations) against the dislocations inside whisker are assumed to exist at mirror-symmetry positions with respect to the whisker surface. The dislocations inside whisker and the image dislocations would attract with each other (hereafter, 'the attractive force' is referred to as image force), and they tend to combine with each other and disappear at the whisker surface. Therefore, the dislocations within whisker would move toward the whisker side-surface, resulting in the inducement 
of dislocation bending inside the whisker and the dislocation intersects with the side-surface of whisker, forming slip bands on the side-surface of whisker [13]. The dislocations tend to become normal to the side-surface of whisker to decrease dislocation energy as shown in Fig. 5.

The bent dislocations in Fig. 5 move also by the image force, and this conservative motion of dislocations

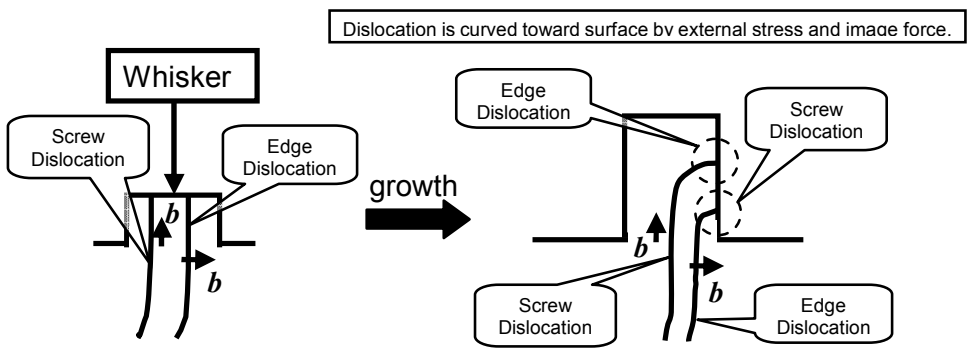

Fig. 5 Dislocations inside whisker during growth process of whisker. $\boldsymbol{b}$ is the Burgers vector. induces the deformation of whisker without the change in C.O. Furthermore, as shown in Fig. 6 , dislocations with same sign of $\boldsymbol{b}$ would accumulate nearly on one crystallographic plane. In the case of the accumulated dislocations being edge dislocations, small-angle tilt boundary (SATB) would be formed, accompanying the small change in C.O. in the regions between the SATB. It is possible that the accumulation of both edge and screw dislocations along different directions forms small-angle boundaries (SAB) with the change in C.O. in the regions between SAB. The change in the shape or bending of whisker with or without the change in C.O. would depend on both the configuration and arrangement of the dislocations. Figures 7 and 8 are the UHVEM micrographs taken from a whisker showing a SAB (indicated by arrows) and equi-thickness fringes of both sides of the $\mathrm{SAB}$, respectively, showing the difference of crystallographic orientation (DCO) between the both sides of the SAB. The difference between the distances of equi-thickness fringes corresponds to the DCO.

It is often observed that a whisker bends more than two times after long interval, suggesting that recovery due to Sn diffusion would be necessary to release the tangle of dislocations which are epitaxially inherited from the piled-up dislocations by deformation in bulk Sn. After the recovery, dislocations would become mobile by both the external shear stress and the image force. For example, in addition of the effects of the external shear stress and the image force, the formation of helical dislocations due to the reaction of $\mathrm{Sn}$ atoms with jogs of screw dislocation could occur, and the helical dislocations with edge component could form SATB through the elastic interaction among them, inducing the small amounts of change in C.O. as shown in Fig. 9.

The bending of dislocations changes their natures, namely, screw and edge dislocations bent near the whisker side-surface change into edge and screw dislocations, respectively (Fig.5). This phenomenon could induce the whisker growth from its root [9].

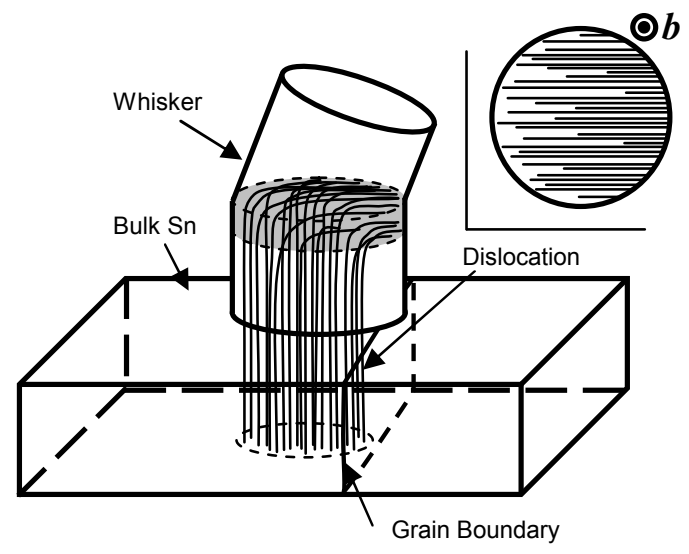

Fig. 6 An example of formation of a small angle boundary due to two-dimensional configuration of edge dislocations. The upper-right inset is a cross-sectional view of the shaded part. $\boldsymbol{b}$ is the Burgers vector.

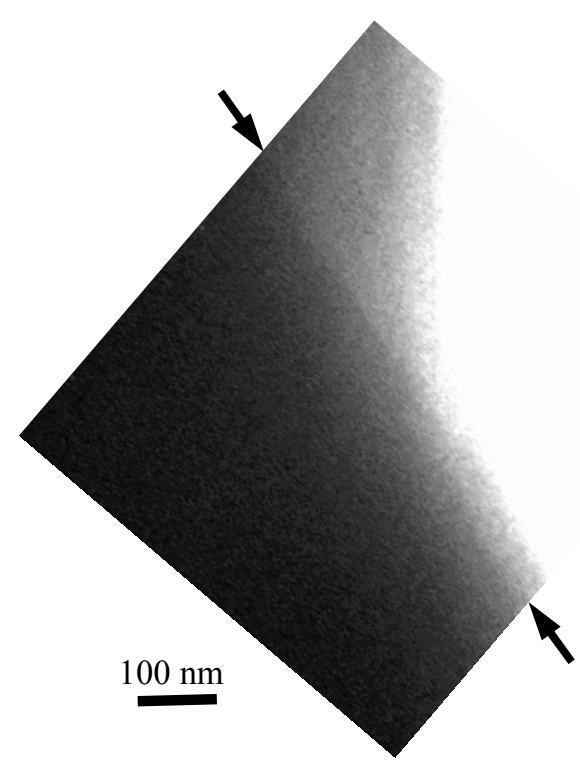

Fig. 7 UHVEM micrograph showing smallangle dislocation boundary (SAB). As the thickness of whisker is large, about $2 \mu \mathrm{m}$, dislocations on the SAB could not be observed, but the conditions of Bragg reflection are slightly different between the SAB, inducing the different brightness (see also Fig. 8). 
Migrating Sn atoms in bulk Sn are supplied into whisker though the pipe diffusion only along edge dislocation, but Sn atoms scarcely migrate along screw dislocation which is formed by the bending of edge dislocation. Sn atoms supplied as jogs of edge dislocation would be released from the edge dislocation around its bending area where edge dislocation changes to screw one. In order to lower the edge dislocation energy which increases by the jogs of Sn atoms, namely, the increase in dislocation length, the jogs of Sn atoms would easily dissociate from the edge dislocation, resulting in diffusion of $\mathrm{Sn}$ atoms into whisker [13]. Concentration of vacancies in whisker would be lower than that in bulk Sn, because a unit volume of whisker has more amount of surface where vacancies disappear than a unit volume of bulk $\mathrm{Sn}$ has. Then, vacancy migrates into whisker along the concentration gradient of vacancies. $\mathrm{Sn}$ atoms dissociated from edge dislocations, which are densely accumulated also within whisker by continuous loading (deformation) of bulk Sn, easily diffuse into whisker, and the growth of whisker occurs remarkably near its root. Vacancies would disappear at the edge dislocation (the dislocation becomes straight and short by the absorption of vacancies) by its replacement with Sn jogs at the edge dislocation. The repeat of both the replacement of Sn jogs on edge dislocation and vacancies and the pipe diffusion through new formation of Sn jog on the edge dislocation would induce the growth of whisker near its root. Whisker tends to maintain the shape of cross-section macroscopically in the course of growth. This phenomenon is likely to occur in order to suppress the increase in surface energy, inducing the growth of whisker being enclosed by preferred crystallographic planes.

It could be suggested that the motion of dislocations induces deformation of whisker with or without crystallographic rotation of whisker. The dislocation motion, the elastic interaction between dislocations, the image force or both accumulation and configuration of dislocations in bulk $\mathrm{Sn}$ would induce unexpected change in the shape of whisker.

According to the observation of oxide film on $\mathrm{Sn}$ whisker, the oxide film is the amorphous containing small crystallites of Sn oxide as shown in Fig. 8, also

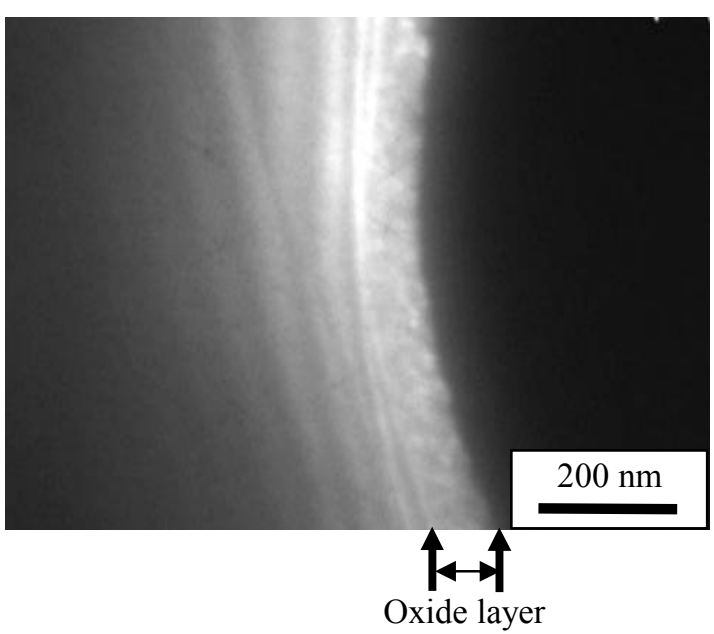

Fig. 8 UHVEM micrograph showing thickness fringes and oxide layer on a whisker. The oxide layer is amorphous containing small crystallites of Sn oxide.
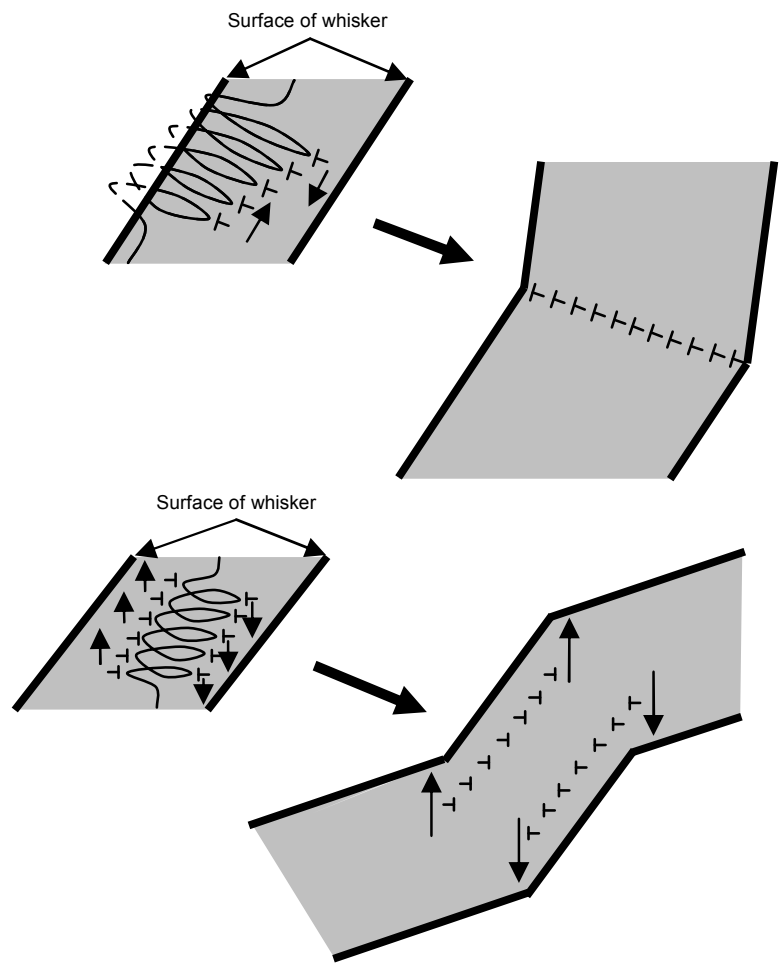

Fig. 9 Examples of the formation of small angle boundary or deformation due to motions of edge dislocations of a helical dislocation. being clarified due to the electron diffraction taken from the oxide layer. It could be suggested that the thickness of the oxide film would not depend on the Sn grain, and the oxide film would not be a controlling for whisker formation. 


\section{Conclusions}

(1) Densely localized screw dislocations would induce the nucleation of whisker, because the trapping sites for Sn atoms migrating on surface are supplied by the screw dislocations.

(2) It would be necessary that both screw and edge dislocations are densely accumulated in the localized region (e.g. around grain boundary) for the nucleation and growth of whisker. The accumulation of dislocations by the operation of various slip systems is indispensable to supply both screw and edge dislocations at a localized region.

(3) Edge dislocations at the same area where dense screw dislocations exist are necessary for supplying $\mathrm{Sn}$ atoms from the bulk Sn into whisker through pipe diffusion along the edge dislocations.

(4) The change in the shape of whisker (e.g. the bending of whisker) would be due to conservative motion of dislocations with maintaining the state of single crystal in whisker and the formation of $\mathrm{SAB}$ with the change in C.O. in the regions across SAB.

(5) Growth of whisker from its root is due to the bending of edge dislocation in whisker by both the external shear stress and the image force. The bending of edge dislocation changes its nature to screw one around the root of whisker. Around the whisker root, Sn atoms accumulated as jogs of edge dislocation by the pipe diffusion would dissociate from the edge dislocation around the bending area of edge dislocation to decrease the edge dislocation energy, namely, shorten the edge dislocation.

(6) It could be suggested that the above mechanism acts also in electroplated $\mathrm{Pb}$-free $\mathrm{Sn}$ layer on $\mathrm{Cu}$ based practical materials, because large stress fields exist also in the electroplated Sn layer.

\section{Acknowledgements}

Authors would like to express sincere thanks to the Small and Medium Enterprise Agency, the Ministry of Economy, Trade and Industry, Japan for providing the Research Grant-in-Aid for the promotion of this research.

\section{References}

[1] K. Murakami, M. Hino, M. Takamizawa, K. Nakai, J. Japan Inst. Metals 72 (2008) 168-175.

[2] Y. Nakadaira, S. Jeong, J. Shim, J. Seo, S. Min, T. Cho, S. Kang, S. Oh, Microelectronics Reliability 48 (2008) 83-104.

[3] K.S. Kim, C.H. Yu, S.W. Han, K.C. Yang, J.H. Kim, Microelectronics Reliability 48 (2008) 111-118.

[4] H. Chuang, H. J. Lin, C. C. Chi, Scr. Mater. 56 (2007) 45-48.

[5] B. Jiang, A.-P. Xian, Philos. Mag. Lett. 87 (2007) 657-662.

[6] T. -H. Chuang, Scr. Mater. 55 (2006) 983-986.

[7] B. Jiang, A. -P. Xian, Philos. Mag. Lett. 86 (2006) 521-527.

[8] K. -N. Tu, J. -O. Suh, A. T. -C. Wu, N. Tamura, C. -H. Tung, Mater. Trans. 46 (2005) 2300-2308.

[9] K.N. Tu, J.C.M. Li, Mater. Sci. Eng. A 409 (2005) 131-139.

[10] S. H. Liu, C. Chen, P. C. Liu, T. Chou, J. Appl. Phys. 95 (2004) 7742-7747.

[11] Y. K. Min, Z. A. Munir, H. Schmaltzried, Philos. Mag. A 71 (1995) 815-829.

[12] A. R. Verma, Nature, 167 (1951) 939.

[13] K. Nakai, T. Sakamoto, S. Kobayashi, M. Takamizawa, K. Murakami and M. Hino, J. Phys. in press.

[14] K. S. Kim, C. H. Yu, J. M. Yang, Microelectronics Reliability 46 (2006) 1080-1086. 